\title{
Proteomic signature of Temporomandibular Joint Disorders (TMD): Toward diagnostically predictive biomarkers
}

\author{
Garabed Gary Demerjian ${ }^{1}$, Anothony Benjamin Sims ${ }^{2}$, Brendan Curran Stack ${ }^{3}$
}

${ }^{1}$ Private Practice, Glendora and Burbank, CA; ${ }^{2}$ Private Practice, Columbia, MD; ${ }^{3}$ Private Practice, Vienna, VA; Garabed Gary Demerjian - Email: drdemerjian@yahoo.com; *Corresponding author

\begin{abstract}
:
The temporomandibular joint (TMJ) articulates the mandible with the maxilla. Temporomandibular joint disorders (TMD) are dysfunctions of this joint, which range from acute to chronic inflammation, trauma and dislocations, developmental anomalies and neoplasia. TMD manifest as signs and symptoms that involve the surrounding muscles, ligaments, bones, synovial capsule, connective tissue, teeth and innervations proximal and distal to this joint. TMD induce proximal and distal, chronic and acute, dull or intense pain and discomfort, muscle spasm, clicking/popping sounds upon opening and closing of the mouth, and chewing or speaking difficulties. The trigeminal cranial nerve V, and its branches provide the primary sensory innervation to the TMJ. Our clinical work suggests that the auriculotemporal (AT) nerve, a branch of the mandibular nerve, the largest of the three divisions of the trigeminal nerve, plays a critical role in TMD sequelae. The AT nerve provides the somatosensory fibers that supply the joint, the middle ear, and the temporal region. By projecting fibers toward the otic ganglion, the AT nerve establishes an important bridge to the sympathetic system. As it courses posteriorly to the condylar head of the TMJ, compression, injury or irritation of the AT nerve can lead to significant neurologic and neuro-muscular disorders, including Tourette's syndrome,Torticolli, gait or balance disorders and Parkinson's disease. Here, we propose that a proteomic signature of TMD can be obtained by assessing certain biomarkers in local (e.g., synovial fluid at the joint) and distal body fluids (e.g., saliva, cerebrospinal fluid), which can aid TMD diagnosis and prognosis.
\end{abstract}

\section{Background:}

Neurologic disorders, including dystonias, and Parkinson's disease, are common in aging adults. Treatments vary, but evidence-based and comparative effectiveness analysis has not yielded systematically reviewed best available evidence of satisfactory interventions. The auriculotemporal (AT) nerve, a branch of the mandibular nerve, innervates the temporomandibular joint, and courses to the tympanic membrane and anterior cochlear surface and neighboring tissues. AT fibers project to the sympathetic otic ganglion. Clenching, grinding, trauma, bone loss and stress can change the jaw bite, and decrease its vertical dimension. Subsequent irritation and compression of the AT nerve can occur, with associated parasthesia, pain and discomfort. Symptoms can be local and specific (e.g., TMD), as well as varied and systemic (e.g., neurologic, dystonic and neuro-muscular disorders, including tremors, muscle spasms leading to impaired and awkward positional control of the head, hands, other extremities, speech impairment, incontinence, impaired sleep, associated depressive symptomatology).

Diagnosis of TMD, for example by comparing the temporomandibular joint space and condylar position by meticulous scanning of tomographic or cone beam projections in patients with anterior disc displacement, is challenging. Managing patients with TMD is also a complex endeavor [1].

We have observed that intervention, aimed at changing the maxillomandibular occlusal relationship by changing the vertical dimension of the patient's jaw bite, relieves AT compression and associated irritation, and leads to immediate and sustained symptomatic improvement clinically (Figure 1). A profile of certain proteomic biomarkers of pain and of temporomandibular joint dysfunction may be assembled to assess and detect the prognostic changes in TMD following ISSN 0973-2063 (online) 0973-8894 (print) Bioinformation 5(7): 282-284 (2011) intervention directed specifically at relieving the irritation and compression of the AT nerve.

Radiographic, arthrotomographic and histologic investigations have established the anatomic relationship between the joint components and the different nerves running in the vicinity of the joint. In several instances, the AT nerve had its course between the condyle and the temporal bone glenoid fossa wall, exposing it to the risk of mechanical irritation during medial disc displacement. These findings may explain the pain associated with the joint movements, and other sensations projecting to the terminal area of distribution of the nerve branches in the vicinity of the joint, such as the ear, temple, cheek, tongue, and teeth, and perhaps more distally [2] Systematic reviews of the best available evidence indicate that hard stabilization appliances, when adjusted properly, have good-to-modest efficacy in treating TMD pain, compared to non-occluding appliances. Hard appliances may have increased effectiveness and reduced risk for potential for adverse events [3]. Our hypothesis also rests on the anatomical observation that the AT nerve is repeatedly and positively identified in every specimen examined on each side, as a single trunk coursing along the medial aspect of the condylar neck. At the posterior border of the lateral pterygoid muscle, the nerve trunk is in direct contact with the condylar neck, thus being at risk of injury as occlusion of the temporomandibular joint is altered by bone loss, trauma, grinding and other pathological etiologies [4]. Indeed, in most cases, the AT nerve is 10$13 \mathrm{~mm}$ inferior to the superior surface of the condyle and 1-2 mm posterior to the neck of the condyle, thus in an anatomical location that position that is at high risk for entrapment with adjacent tissues [5]. Moreover, the anatomical relationship of the AT nerve course and the superficial temporal artery was proposed to contribute to explaining the source of 
migraine headaches and other referred pains in certain patients with TMD [6].

Systemic exacerbations of pathologies of the AT branch of the trigeminal nerve can arise as manifestations of the trigeminal reflex. Case in point, the trigemino-cardiac reflex presents as parasympathetic dysrhythmia, sympathetic hypotension, apnea, or gastric hyper-motility, consequential to the stimulation of any of the sensory branches of the trigeminal nerve. The proposed mechanism of this response involves the Gasserian ganglion, which ochastrates the mechanical stimulation of all the central and peripheral branches of the trigeminal nerve. Remarkably patients may develop severe bradycardia, asystole, and arterial hypotension, and other symptoms which subside almost immediately with cessation of the stimulus [7, 8]. The loops of this reflex involves multisynaptic neck muscle withdrawal responses, mediated by neural circuits at brainstem level, and absent or impaired in patients with Parkinson's disease or progressive supranuclear palsy, putatively because of a degeneration of brainstem neural structures $[9, \mathbf{1 0}]$.

In brief, we propose that markers may be tested in aspirates of the synovial fluid at the temporomandibular joint for local assessment, as well as in whole or parotid saliva, plasma and cerebrospinal fluid for distal and systemic measurements of AT pathology. We suggest that a carefully assembled proteomic biomarkers will be beneficial in supplementing the clinical imaging profile provided by cone beam Computed Tomography (cb-CT), which will provide a representation of the structural alterations in TMD, and Positron emission tomography (PET) to yield a threedimensional image of the functionality of the joint, as well as its associated musculature and neurological processes centrally and peripherally.

\section{Methodology:}

Admitted patients provide a thorough clinical history of symptoms and chief complaints, including allergies to medications, current medications being taken, and other physicians consulted. Photos of their posture with a posture grid, close up photos of the head and shoulders with and withou a tongue blade between the teeth, to evaluate a cant of the maxilla are taken. Intra-oral photos are also taken. The physical examination consists of palpations of the head, neck, facial and intraoral muscles for evaluation and diagnoses. The mandibular range of motion serves to establish deviations of deflection of the jaw while opening and closing. Clicking, popping or lack of translation of the joints are noted. The intraoral examination serves to establish signs of infection, decay, and oral cancer. Signs of bruxism are noted, evident through presence of occlusal wear patterns, abfractions, recessions at the bicuspid areas, loss of vertical dimension, missing teeth, tori, and dental midlines deviation while closed. Cervical range of motion is measured: extension, flexion and rotation are noted. Skeletal morphology and posture is evaluated for facial asymmetry, including forward head position, kyphotic cervical spine and difference in shoulder heights.

Computerized testing of the muscles of mastication followes, and electrognathologic assessments measure and document jaw mobility. A velocity test measures how fast the jaw moves to evaluate any restrictions of muscular movements in the opening and closing of the jaw. Join vibration analysis evaluation allows listening and measuring of any join sound, what type and at what point the sound occurs, such as an early opening click or grating sound like crepetus (bone on bone rubbing). The physiologic properties of the masticatory muscles (left and right anterior temporalis, masseters, anterior digastrics, and sternocleidomastoids) are obtained by electromyography (Figure 1)

When the patient suffers movement disorder (e.g., facial tics, tremors, or improper posture, dystonia), the maxillomandibular vertical dimension is opened at $2 \mathrm{~mm}$ increments by placing tongue blades between the teeth to obtain a normal physiological maxillomandibular relationship, which is observed and/or achieved when symptoms and dysfunctions disappear or are greatly improved.

We hypothesize that the levels and concentrations of certain pain mediators (e.g., Substance P), catecholamines (e.g., norepinephrine), pro- inflammatory cytokines (e.g., interleukin [IL]-1b, IL-6, IL-10, tumor necrosis factor, monocyte chemoattractant protein-1 [11], and soluble cytokine receptors [12] can be obtained locally in synovial fluid from patients with different stages of TMD, as well as distally (e.g., parotid and whole saliva) and systemically (e.g., plasma, cerebrospinal fluid). These measurements will be correlated with the covariate of synovial lubricin and hyaluronic acid concentrations, which has been used as a staging indicator of TMD [13]. Markers will be assessed by enzyme-linked immunosorbent assay (ELISA), radio-immune assays (RIA), or Surface-enhanced laser desorption/ionization (SELDI) or matrix-assisted laser desorption/ionization (MALDI), as required. The proteomic profile will be correlated with the clinical observations, imaging (i.e., cb-CT, PET), and cumulative neuroendocrine assessments of chronic pain stress, such as what can be obtained by measurement of cortisol levels in hair shaft [14].

\section{Discussion:}

The proteome represents the entire complement of proteins, including epigenetic alterations, that characterize a physiocellular site, organ or organism. The proteome is a set of characteristics that determines and defines the physio-pathological, biochemical and molecular state of a system within the organism, and of the organism at large. The proteomic profile changes and varies with time and age, and adapts to the distinct demands and requirements of the environment, during the lifetime of the organism.

The normal physiological proteome of, for example, the temporomandibular joint represents the set of proteins and molecules expressed by the cells in that anatomical locus, and involved in its normal anatomical functioning. The proteomic signature of the healthy joint is distinctively different from what is observed in the pathological conditions of TMD, where pain is expressed and identified biologically by pain transmitters, inflammation is observed and recorded as pro-inflammatory agents, and fundamental lubricants of a well-functioning joint decrease or are absent. A variety of sensitive, reliable and valid techniques are now commercially available to ensure the replicable assessment and monitoring of such factors. Methodologies such as ELISA, RIA, SELDI and MALDI - the latter usually tested in conjunction with time-of-flight (TOF) mass spectrometry (hence, SELDI-TOF and MALDI-TOF) - are now routine in most laboratories specialized in the detection of proteomic signature [15, 16].

Structural and functional imaging techniques (e.g., cb-CT, PET), while costly and requiring sophisticated clinical equipment, yield important clinical information about TMD. Expectations are that the proteomic signature we propose here will be strongly correlated with the imaging data. The proposed proteomic approach will also be less taxing to the patients than the imaging protocol because the process of obtaining body fluids, such as blood for isolation of plasma, and whole saliva are routine and associated with minimal discomfort and risk.

Obtention of parotid saliva through the Stensen duct is a bit more uncomfortable for the patient, but it has the advantage of producing a body fluid that is considerably more pure than whole saliva, which can be contaminated by plasma emanating from bleeding gums or oral ulcers. Collection of synovial fluid from the capsule of the temporomandibular joint can be painful to the patient, and suffers from the disadvantage of producing only trace amounts of fluid. However, SELDI and MALDI require only very microliter amounts of sample, thus this limitation is not prohibitive.

Synovial fluid has the superlative advantage of providing real-time changes in the proteomic signature of the joint as our intervention of increasing the bite vertical dimension aimed at decreasing the compression and irritation of the AT nerve immediately relieves the response of the Gasserian ganglion (i.e., the trigeminal ganglion deep within the dura mater, near the apex of the petrous part of the temporal bone), as noted above. The systemic distal equivalent may be obtained by assessments obtained from the cerebrospinal fluid, which may be an equally disturbing, invasive and painful clinical procedure for the patient. 
Whether it is a disc disorder or a mal-relationship between the mandibular condyle and the cranial base, we believe that there is a light pressure or irritation of the auriculotemporal nerve which is sending impulses through the trigeminal system to the brain stem. As the trigeminal enters the brain stem at the pons, sensory fibers go to the medulla and the spinal cord. In the nucleus caudalis of the trigeminal system of the spinal cord are crossover fiber connections of other cranial nerves as they pass by [17] The proper maxillomandibular relationship affects this complex system. When the trigeminal signals are produced properly it effects all the cross over fibers of the brain stem, thus causing the changes/improvements measured in movement disorder patients (i.e., tics and tremors decrease or stop immediately, correcting of the head posture or position, release of tension in the face, strengthening of upper body, gait and balance improvement, and release of neck tension) [18].

At present, PET, which requires a radioactive tracer (i.e., 2-deoxy-2$\left({ }^{18} \mathrm{~F}\right)$ fluoro-D-glucose, a glucose analog with the positron-emitting radioactive isotope fluorine-18 substituted at the 2' hydroxyl position of glucose), with consequential limitations of prohibitive cost, intravenous injection risks, and patient reactivity, is the only clinical protocol available to obtain functional metabolism of central and peripheral neurological activity. Cb-CT yields accurate anatomic information in TMD, but subjects the patients to X-ray, and thus should be used sparingly.

However delicate, the collection of the body fluids proposed here will be informative, we predict, by providing a proteomic signature in healthy and diseased joint that will complement the diagnosis obtained by clinica examination. The levels of the proband biomarkers in each of these fluids will be specifically indicative of the course of the resolution of TMD following our intervention directed at opening the bite, and decompressing the AT nerve. The proteomic signature will be complementary to, and predictive of the imaging data.

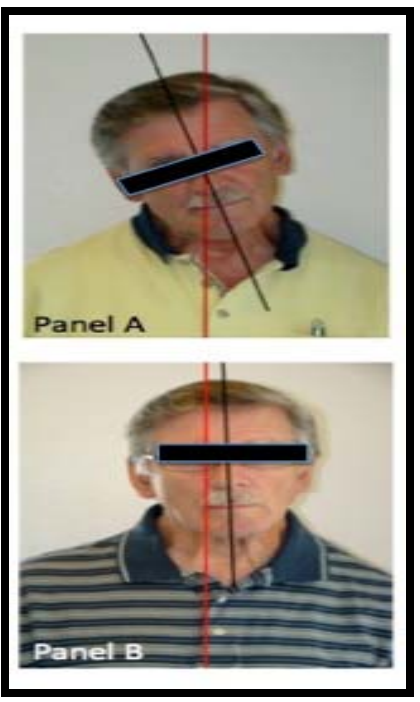

Figure 1: (A) Before Treatment; (B) Immediately after delivery of Intraoral Appliance

Conclusion:
From a clinical standpoint, the evidence is mounting in support of the notion that several of the patients we see with neurological disorders, including Torticolli and Parkinson's disease, dystonia and even Tourette's syndrome and Tics may actually be suffering from TMJ issues. Our clinical observation supports the hypothesis that this group of patients may be suffering of undiagnosed TMJ disc dislocation, subsequent distal condylar displacement and associated compression and irritation of the AT nerve. We have shown that by treating the TMD and making changes in the maxillomandibular relationship with some type of a hard intraoral appliance or a neurocranial vertical distractor (NCVD) [18], we are not only effectingand treating the symptoms of pain and discomfort, but we also resolve symptoms of neurologic disorders, neuromuscular dysfunctions, and dystonia. Clinically, this is accomplished simply by removing the irritation or compression of the AT nerve, which serves the joint disc and capsule.

With the help of the biomarkers, we will be able to monitor the physiological changes being seen clinically such as the cessation or reduction of tics and tremors, improvements of gait, improvements in head posture and decrease in facial tension. The proteomic signature of TMD, obtained by assessing certain biomarkers in local and distal body fluids, will aid TMD diagnosis, prognosis and treatment. It will yield measurable outcomes of the progress of treatment aimed at returning the nervous system to normal function, and not be overloaded with noxious stimulation. This will be achieved by reliable molecular biological protocols, and minimal risk and discomfort to the patient. The assessment of proteomic signatures, while somewhat costly, is considerably more costeffective, as informative clinically, and less biohazardous than imaging techniques available presently (e.g., cb-CT, PET).

References:

[1] CS Greene. Am J Orthod Dentofacial Orthop 138: 3 (2010) [PMID: 20620818]

[2] AS Johansson et al. J Oral Maxillofac Surg. 48: 953 (1990) [PMID: 2395048]

[3] J Fricton et al. J Orofac Pain 24: 237 (2010) [PMID: 20664825]

[4] BL Schmidt et al. Oral Surg Oral Med Oral Pathol Oral Radiol Endod 86: 165 (1998) [PMID: 9720090]

[5] PR Fernandes et al. Cranio 21: 165 (2003) [PMID: 12889671]

[6] JE Janis et al. Plast Reconstr Surg 125: 1422 (2010) [PMID: 20440161]

[7] B Arasho et al. Neurol India 57: 375 (2009) [PMID: 19770535]

[8] B Schaller et al. J Neurosurg Anesthesiol 21: 187 (2009) [PMID: 19542994]

[9] M Bartolo et al. Mov Disord 23: 1475 (2008) [PMID: 18561341]

[10] M Aramideh \& BW Ongerboer de Visser. Muscle Nerve 26: 14 (2002) [PMID: 12115945]

[11] N Ogura et al. J Dent Res. 2010 Jul 20. [Epub] [PMID: 20647497]

[12] K Kaneyama et al. J Oral Maxillofac Surg 68: 1088 (2010) [PMID: 20149511]

[13] L Wei et al. J Oral Maxillofac Surg 68(10): 2478 (2010) [PMID: 20678837]

[14] SH Van Uum et al. Stress 11: 483 (2008) [PMID: 18609301]

[15] F Chiappelli et al. Bioinformation 1: 331 (2006) [PMID: 17597915]

[16] OO Oluwadara \& F Chiappelli. Bioinformation 3: 332 (2009) [PMID: 19707295]

[17] AB Sims \& BC Stack. Cranio 27: 11 (2009) [PMID: 19241794]

[18] BC Stack \& AB Sims. Cranio 27: 248 (2009) [PMID: 19891259]

Edited by F Chiappelli

Citation: Demerjian et al. Bioinformation 5(7): 282-284 (2011)

License statement: This is an open-access article, which permits unrestricted use, distribution, and reproduction in any medium, for non-commercial 